\title{
Temperature Dependent Variations of Phonon Interactions in Nanocrystalline Cerium Oxide
}

\author{
Sugandha Dogra Pandey, Jasveer Singh, K. Samanta, \\ Nita Dilawar Sharma, and A. K. Bandyopadhyay \\ Pressure Standards and Pressure Physics, National Physical Laboratory, Dr. K. S. Krishnan Road, New Delhi 110012, India \\ Correspondence should be addressed to Nita Dilawar Sharma; ndilawar@nplindia.org
}

Received 15 January 2015; Revised 1 April 2015; Accepted 9 April 2015

Academic Editor: Ion Tiginyanu

Copyright (c) 2015 Sugandha Dogra Pandey et al. This is an open access article distributed under the Creative Commons Attribution License, which permits unrestricted use, distribution, and reproduction in any medium, provided the original work is properly cited.

\begin{abstract}
The temperature dependent anharmonic behavior of the phonon modes of nanocrystalline $\mathrm{CeO}_{2}$ was investigated in the temperature range of $80-440 \mathrm{~K}$. The anharmonic constants have been derived from the shift in phonon modes fitted to account for the anharmonic contributions as well as the thermal expansion contribution using the high pressure parameters derived from our own high pressure experimental data reported previously. The total anharmonicity has also been estimated from the true anharmonicity as well as quasiharmonic component. In the line-width variation analysis, the cubic anharmonic term was found to dominate the quartic term. Finally, the phonon lifetime also reflected the trend so observed.
\end{abstract}

\section{Introduction}

$\mathrm{CeO}_{2}$ is a strategic material which has been extensively investigated and has recently been found to be of use in intermediate temperature oxide fuel cells as it easily absorbs and releases oxygen [1]. It also shows enhanced ionic conductivity with temperature [2]. Hence, the investigation of the temperature dependent behavior of $\mathrm{CeO}_{2}$ has gained immense interest. Under ambient conditions, it usually crystallizes in the cubic fluorite structure with space group Fm3m [3]. Nanocrystalline cerium oxide has applications in the coating technology, for example, wood-coating industry [4].

It is well known that an increase in temperature in any material introduces perturbations in the harmonic potential term, which changes the vibrational properties. Temperature affects the population of the different levels for each normal mode instigating the change of the lattice parameters. Any change of the lattice parameters with temperature is attributed to the anharmonicity of the lattice potential. As an effect of the anharmonic interaction, a nonequilibrium phonon population decays into phonons of lower energy or is scattered by thermal phonons into modes of frequencies shifted from their original positions [5]. These anharmonic interactions occur primarily due to the cubic or quartic anharmonicities, resulting in the decaying of an optical phonon into two or three acoustic phonons, respectively, which also decreases their lifetimes. As a consequence, the phonon line position and bandwidth in the Raman spectra of various amorphous or crystalline materials exhibit significant changes with temperature [6-8]. Further, the finite-size effects in nanocrystals are expected to modify the anharmonicity and the phonon decay times. It has also been reported that the anharmonic constants related to the peak widths and peak positions are higher in nanocrystals than in the bulk, implying a greater degree of anharmonicity in nanocrystals [9]. Very few experiments have been carried out to investigate the temperature dependence of Raman mode frequencies and line-widths in nanocrystals [5, 10-12].

Still fewer studies have been carried out on the anharmonic behavior of the $\mathrm{CeO}_{2}$ phonon modes [13-15]. Popović et al. [13] reported that three contributions are involved in the temperature-induced Raman mode frequency shifts of nano- $\mathrm{CeO}_{2-y}$ : the thermal expansion, the anharmonic effect, and the phonon frequency change due to the change of nanoparticle size with temperature (confinement effect). It is natural to expect that in nanomaterials the contribution of four-phonon processes should be larger compared to that of the three-phonon processes [11]. However, Spanier et al. [14] 
reported that phonon coupling is not stronger in the $\mathrm{CeO}_{2-y}$ nanoparticle system than that in the bulk counterpart. Consequently, the aim of this work is to examine the temperatureinduced changes in the vibrational properties of $\mathrm{CeO}_{2}$, to identify the contribution of volume expansion and anharmonic intermode coupling to the Raman frequency shift, line-width, and the phonon lifetime in $\mathrm{CeO}_{2}$.

In this context, we report the temperature dependent Raman behavior of nanocrystalline $\mathrm{CeO}_{2}$ and its anharmonic constants derived using our previously published high pressure data [16]. We have analyzed the softening of the most dominant mode $\left(F_{2 g}\right)$ in light of the theory of anharmonic phonon-phonon interaction and thermal expansion of the lattice.

\section{Characterization at Ambient Conditions}

The $\mathrm{CeO}_{2}$ powder used in the present study is a commercially prepared high purity powder from Johnson Matthey Rare Earth Products, UK. The powder was used in the present study in as-received state and was not subjected to any pretreatment and, as per the manufacturer's claims, the common metal impurities are at a ppm level. The characterization of this $\mathrm{CeO}_{2}$ nanopowder under ambient conditions via X-ray diffraction, atomic force microscopy, and Raman spectroscopy has been discussed in detail elsewhere [17]. In brief, the crystalline phase at ambient was found to be cubic. The crystallite size of this cubic $\mathrm{CeO}_{2}$ was found to be $36 \mathrm{~nm}$ calculated using Debye-Scherrer relation and about $32 \mathrm{~nm}$ from atomic force microscopy. The predominant Raman peak of $\mathrm{CeO}_{2}$ was obtained at $465 \mathrm{~cm}^{-1}$, assigned as $F_{2 g}$ mode [17]. In Ceria, the first order Raman active mode is the triply degenerate symmetrical stretching vibration of the $\mathrm{CeO}_{8}$ vibrational unit. In bulk $\mathrm{CeO}_{2}$, there is a single allowed Raman active triply degenerate $F_{2 g}$ mode centered at about $465 \mathrm{~cm}^{-1}$. This mode can be viewed as a symmetric breathing vibrational mode of the $\mathrm{O}$ ions around each cation $[18,19]$. The $\mathrm{CeO}_{2}$ belongs to space group Fm3m (225). This structure has two BCC unit cells and has two acoustic modes and 1 optical mode.

\section{Temperature Dependent Raman Studies}

The Raman spectra were recorded in the temperature range $80-440 \mathrm{~K}$. The spectra were acquired using Jobin Yvon T64000 triple Raman spectrometer with the $514.5 \mathrm{~nm}$ line of the Ar ion laser as the source radiation. The sample temperature was varied using a continuous flow liquid nitrogen cryostat from Janis, model ST-500.

The temperature dependent Raman spectra of $\mathrm{CeO}_{2}$ are given in Figure 1. The spectra show changes in line position, line-width, and intensity with an increase in temperature from $80 \mathrm{~K}$ to $440 \mathrm{~K}$. We have focused on temperature dependence of Raman frequency and line-width of the predominant optical $F_{2 g}$ phonon in the studied temperature range. With temperature increase, the $F_{2 g}$ mode shifts to lower frequencies and the line-width continuously increases.

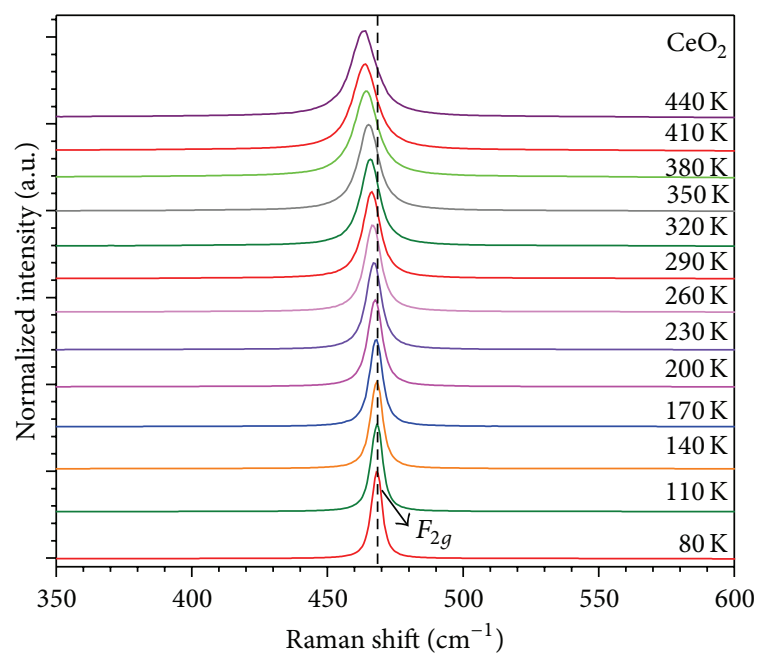

Figure 1: Temperature dependent Raman spectra of $\mathrm{CeO}_{2}$ from $80 \mathrm{~K}$ to $440 \mathrm{~K}$.

A vertical line on the $F_{2 g}$ mode in Figure 1 facilitates the visualization of the extent of shift in said phonon mode.

Hence, the observed results show temperature dependent phonon softening of $F_{2 g}$ mode, that is, red shift with progression of temperature. The possible reasons for red shift can be heating and phonon interactions and so forth. Heat causes expansion of the material which leads to frequency decrease. The frequency softening is significant up to a temperature of $\sim 380 \mathrm{~K}$. Above this temperature, the phonon softening is seen to slow down. It may be mentioned here that, as per the reported Debye temperature of $355 \mathrm{~K}[20,21]$, the phonon softening may be said to be slowing down above the Debye temperature. This may be owing to the fact that, above the Debye temperature, the change in specific heat capacity becomes negligible. Figure 2 shows the $F_{2 g}$ peak positions observed at $80 \mathrm{~K}$ and $440 \mathrm{~K}$ which are centered at $468.2 \mathrm{~cm}^{-1}$ and $463.3 \mathrm{~cm}^{-1}$, respectively. The frequency of this mode can be clearly seen to shift to lower energy side and the line-width increases with temperature. In the studied temperature range, the total quantitative softening of the $F_{2 g}$ mode amounts to about $5 \mathrm{~cm}^{-1}$. These shifts as well as the line-widths estimated from the "peakfit" software are graphically depicted clearly in Figure 3. It may be interesting to note here that similar to the behavior shown by mode softening the line-width increase also shows a lower slope above about $380 \mathrm{~K}$.

The observed phonon softening of the $F_{2 g}$ mode is examined from the theory of anharmonic phonon-phonon interaction and thermal expansion of the lattice. As mentioned, the variation of the frequency of the normal modes with temperature at constant pressure arises from two contributions: pure volume contribution $(\Delta \omega)_{\text {latt }}$ which results from thermal expansion and pure-temperature contribution $(\Delta \omega)_{\text {anh }}$ which results from cubic and quartic anharmonicities (self-energy shift). The cubic and quartic anharmonicities are described by factors which include contributions from three- and four-phonon anharmonic processes [11]. The quasiharmonic 


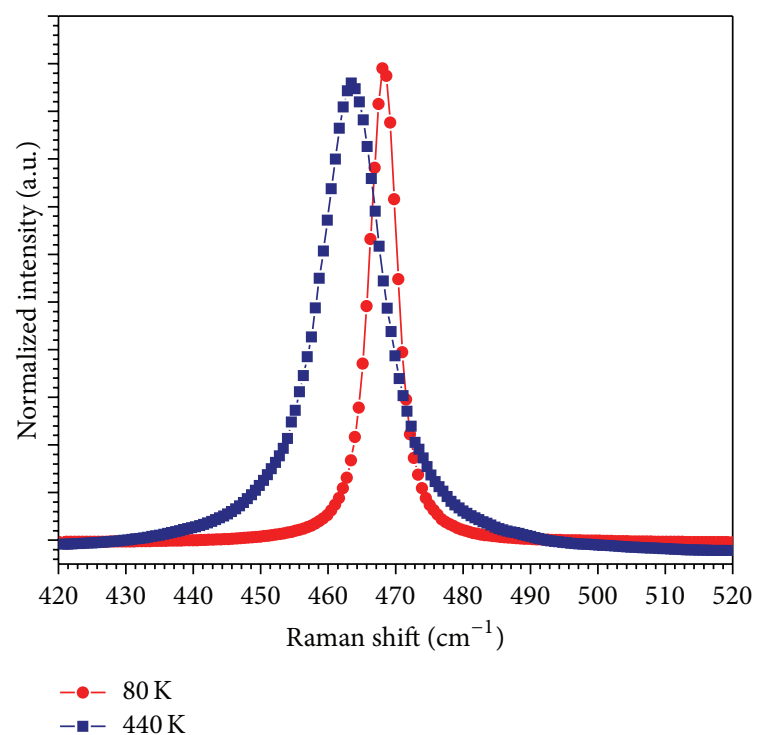

Figure 2: Comparison of the line profile of $F_{2 g}$ mode as a function of temperature at $80 \mathrm{~K}$ and $440 \mathrm{~K}$.

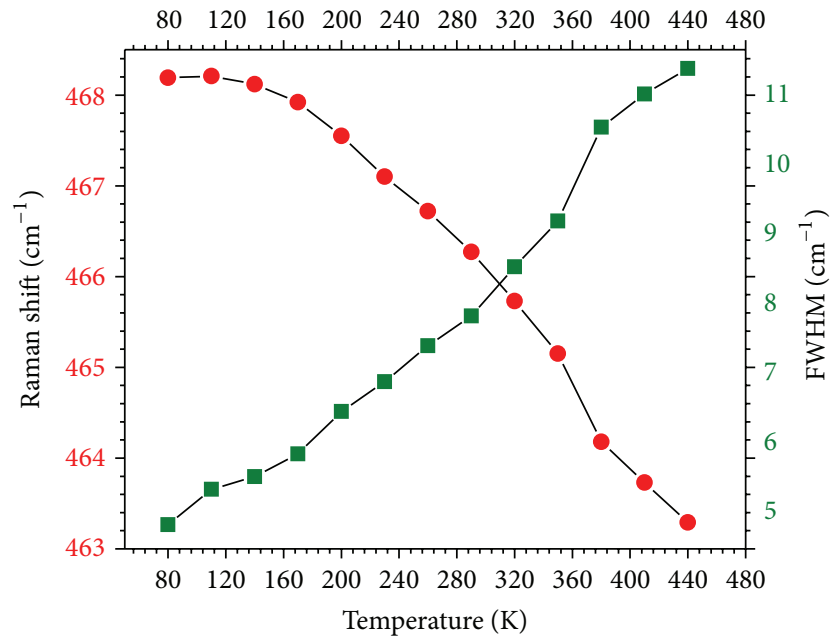

Figure 3: Temperature dependent Raman shift and FWHM of the $F_{2 g}$ mode of $\mathrm{CeO}_{2}$.

term $(\Delta \omega)_{\text {latt }}$ arises from the thermal expansion, while puretemperature contribution $(\Delta \omega)_{\text {anh }}$ due to cubic and quartic anharmonicities is also termed as intrinsic (true) anharmonicity $[9,12]$. Hence,

$$
\omega(T)=\omega_{0}+(\Delta \omega)_{\mathrm{latt}}+(\Delta \omega)_{\mathrm{anh}} .
$$

The quasiharmonic or thermal expansion contribution can be written as

$$
(\Delta \omega)_{\mathrm{latt}}=\omega_{0}\left\{\exp \left[-\gamma_{i} \int_{0}^{T} 3 \alpha(T) d T\right]-1\right\}
$$

where $\omega_{0}\left(=470.1 \mathrm{~cm}^{-1}\right)$ is the harmonic frequency of $F_{2 g}$ mode which is obtained by extrapolating the experimental data down to $0 \mathrm{~K}$. The Grüneisen parameter $\gamma(=1.28)$ of the corresponding mode is estimated from our high pressure Raman scattering data reported elsewhere [16] and $\alpha$ is the linear thermal expansion coefficient.

The anharmonic contribution $(\Delta \omega)_{\text {anh }}$ which arises from the phonon-phonon interaction due to the cubic and quartic terms in the interatomic potential can be written as

$$
\begin{gathered}
(\Delta \omega)_{\text {anh }}=A\left[1+\frac{2}{\exp \left(\hbar \omega_{0} / 2 k T\right)-1}\right]+B[1 \\
\left.+\frac{3}{\exp \left(\hbar \omega_{0} / 3 k T\right)-1}+\frac{3}{\left(\exp \left(\hbar \omega_{0} / 3 k T\right)-1\right)^{2}}\right] .
\end{gathered}
$$

The first term estimates the coupling of an optical phonon to two low-energy phonons (three-phonon process); the second term is due to the coupling of three phonons (fourphonon process), and $A$ and $B$ are the fitting parameters. We have also calculated the total anharmonicity of $F_{2 g}$ mode as a function of temperature using the following equation [22]:

$$
\left(\frac{1}{\omega_{i}} \frac{d \omega_{i}}{d T}\right)_{P}=\left(\frac{1}{\omega_{i}} \frac{d \omega_{i}}{d T}\right)_{V}-\gamma_{i} \alpha .
$$

The first term of the right hand side describes the true anharmonic contribution and the second quasiharmonic term. As mentioned, the resultant frequency shift may be either positive or negative depending on the relative magnitudes of the anharmonic terms in the interatomic potential. At lower temperatures, the main contribution to the softening is due to the positive quartic anharmonicity.

In Figure 4, we have shown the fitted experimental data points (solid circles) by using (1). The fitting parameters obtained for the present case using the above equations are given in Table 1. From the fitting parameters given in the table, it is clear that coefficients $A$ and $B$ both are negative thus explaining the phonon softening. However, coefficient $A$ dominates over $B$ indicating that three-phonon processes or cubic anharmonicity is contributing significantly to the overall softening observed. It is also seen that the true anharmonic term dominates, albeit slightly, over the quasiharmonic term.

A comparison of these coefficients with those obtained by Dohcevic-Mitrovic et al. [15] and Popović et al. [13] reveals that, in $\mathrm{CeO}_{2-y}$ nanocrystals, although a dominance of fourphonon process was observed in the high temperature range by these researchers, however, we have found significant contribution from three-phonon process to the anharmonicity. Our results differ as we have observed a behavior in nanocrystalline $\mathrm{CeO}_{2}$ which has been reported for polycrystalline $\mathrm{CeO}_{2}$ [13]. It is understood that, in small particles of pure and doped ceria nanocrystals, when size effects have minor impact on Raman modes, four-phonon anharmonic processes prevail $[9,15]$. However, when nanopowdered particles are grown enough, the size effects provoke changes of the anharmonic interactions whereby three-phonon coupling prevails over the four-phonon anharmonic processes.

The line-width of the $F_{2 g}$ mode was also studied as a function of temperature in the $80-440 \mathrm{~K}$ range, which, as expected, increased with temperature as shown in Figure 3. 
TABLE 1: Best-fit values for anharmonic constants and true and quasiharmonic contributions in $F_{2 g}$ mode of $\mathrm{CeO}_{2}$.

\begin{tabular}{lcccccc}
\hline Raman mode & $\begin{array}{c}\omega_{0} \\
\left(\mathrm{~cm}^{-1}\right)\end{array}$ & $\begin{array}{c}A \\
\left(\mathrm{~cm}^{-1}\right)\end{array}$ & $\begin{array}{c}B \\
\left(\mathrm{~cm}^{-1}\right)\end{array}$ & $\begin{array}{c}\frac{d \omega}{d T} \\
\left(\mathrm{~cm}^{-1} \mathrm{~K}^{-1}\right)\end{array}$ & $\begin{array}{c}\left(\frac{1}{\omega_{i}} \frac{d \omega_{i}}{d T}\right)_{P} \\
\left(\mathrm{~K}^{-1}\right)\end{array}$ & $\begin{array}{c}\left(\frac{1}{\omega_{i}} \frac{d \omega_{i}}{d T}\right)_{V} \\
\left(\mathrm{~K}^{-1}\right)\end{array}$ \\
\hline$T_{2 g}$ & 470.1 & -2.626 & -0.496 & -0.014 & $-2.98 \times 10^{-5}$ & $-1.92 \times 10^{-5}$ \\
\hline
\end{tabular}

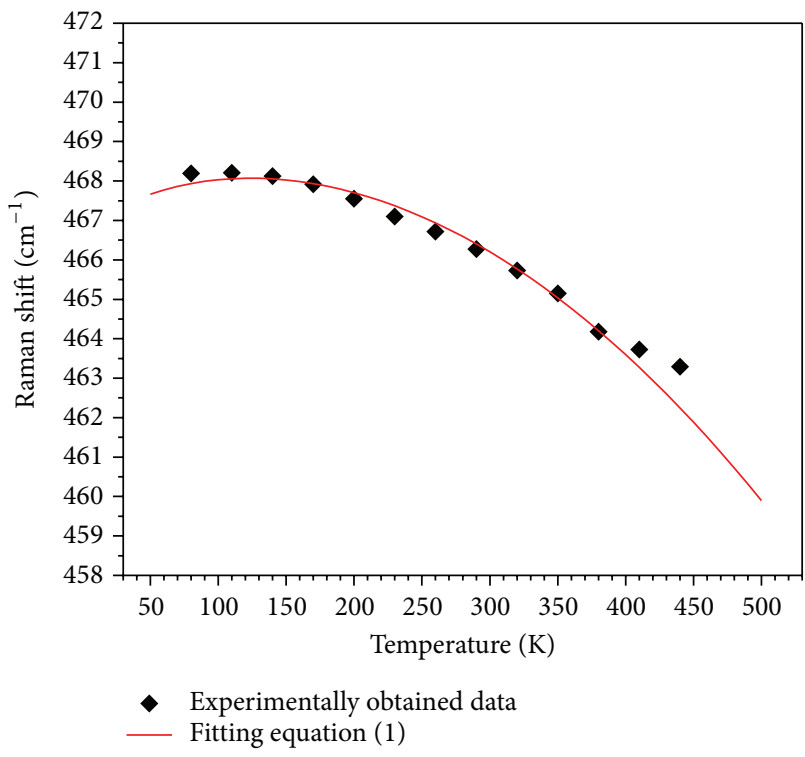

FIGURE 4: Frequency shift of $F_{2 g}$ mode as a function of temperature. The solid line represents the calculated frequency shift using (1).

The FWHM of the $F_{2 g}$ mode increases from $4.85 \mathrm{~cm}^{-1}$ at $80 \mathrm{~K}$ to $11.43 \mathrm{~cm}^{-1}$ at $440 \mathrm{~K}$. Hence, the line-width increases with temperature by about $6.58 \mathrm{~cm}^{-1}$.

The experimental peaks of real materials exhibit an intrinsic width which has contributions from factors such as the finite resolution of the spectrometer and impurities/defects which disturb the translation symmetry of the harmonic crystal $[22,23]$ apart from the anharmonic decay of the phonons. The thermal expansion is a manifestation of the lattice anharmonicity that has its own shifts in the frequency, but not on the line-width [23]. Hence, considering that other effects due to impurities, defects, and spectrometer are constants for a particular sample, the line-width variation would reflect the anharmonic decay of phonons. The temperature dependent phonon line-width is then written as [24]

$$
\begin{gathered}
\Gamma(T)=\Gamma_{0}+C\left[1+\frac{2}{\exp \left(\hbar \omega_{0} / 2 k T\right)-1}\right]+D[1 \\
\left.+\frac{3}{\exp \left(\hbar \omega_{0} / 3 k T\right)-1}+\frac{3}{\left(\exp \left(\hbar \omega_{0} / 3 k T\right)-1\right)^{2}}\right],
\end{gathered}
$$

where $\Gamma_{0}$ is the harmonic line-width independent of temperature and it is caused by defects, including isotopic mixture; the second part of the equation is the damping part induced due to the three- and four-phonon process cubic and quartic anharmonicities, respectively.

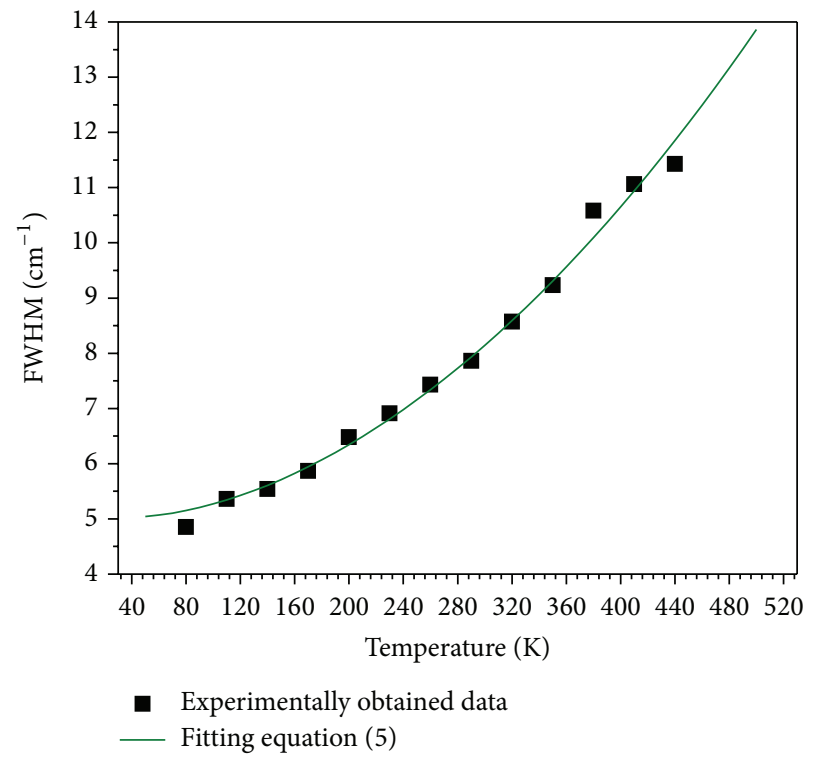

Figure 5: FWHM of $F_{2 g}$ mode as a function of temperature. The solid line represents the calculated FWHM using (5).

Figure 5 shows the fitting to the experimental points using (5). A good agreement can be seen between the calculated curve, given by the solid line, and the experimental points. For the present case, the parameters in (5) are $\Gamma_{0}=2.86 \mathrm{~cm}^{-1}$ and $C$ and $D$ are anharmonic constants where $C=1.65832$ and $D=0.32715$. It may be mentioned here that coefficients $C$ and $D$ are complementary to coefficients $A$ and $B$ which were used to estimate the contribution of anharmonicity to the phonon frequency shift. The positive coefficients describe the increase in line-width with an increase in temperature. The factors calculated using (5) are listed in Table 2.

In this context, it may be pertinent to mention that the Klemens [25] model for the line-width assumed that the contribution to the line-width arises only from the decay of the optical phonon into two acoustic phonons of the same frequency and opposite momentum [3]. Balkanski et al. [11] calculated the change in line-width and line center for silicon using cubic and quartic anharmonicity and found good agreement between theory and experiment. However, it has also been reported that [18] the temperature dependence of the line-width of crystalline $\mathrm{CeO}_{2}$ can be explained completely with consideration of only the cubic anharmonic term of the crystal potential energy and is governed by the phonon occupation number. In our case, although the cubic anharmonic term is predominant, the contribution from four-phonon process is not negligible. 
TABLE 2: Best-fit values for anharmonic constants for $F_{2 g}$ mode of $\mathrm{CeO}_{2}$.

\begin{tabular}{lccc}
\hline Raman mode & $\begin{array}{c}\Gamma_{0} \\
\left(\mathrm{~cm}^{-1}\right)\end{array}$ & $\begin{array}{c}C \\
\left(\mathrm{~cm}^{-1}\right)\end{array}$ & $\begin{array}{c}D \\
\left(\mathrm{~cm}^{-1}\right)\end{array}$ \\
\hline$T_{2 g}$ & 2.86 & 1.65832 & 0.32715 \\
\hline
\end{tabular}

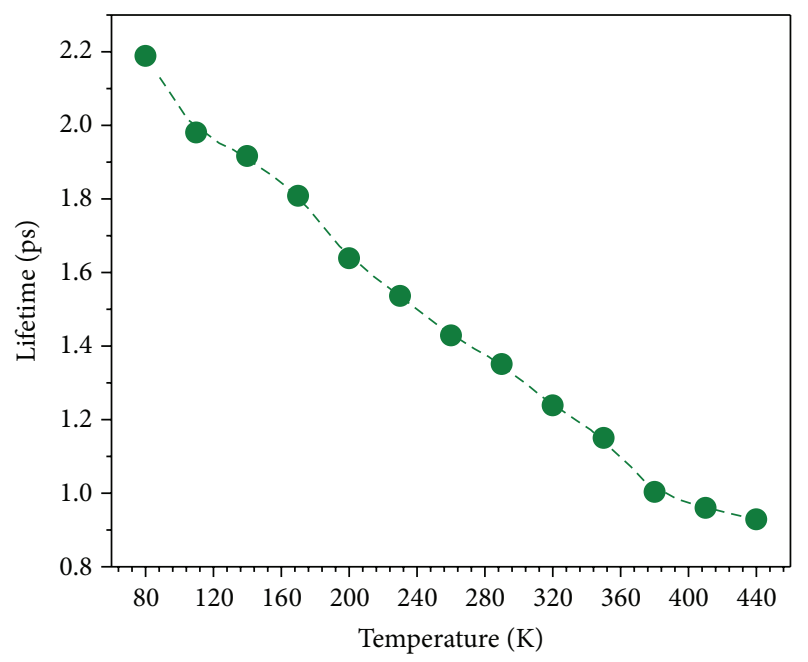

FIGURE 6: Temperature dependent phonon lifetime of $F_{2 g}$ phonon mode.

In addition, changes in the line-width of the phonon in Raman and infrared spectra can be used as an indirect measurement for estimating the lifetime of strongly interacting optical phonons. The line-width of a Raman mode is inversely proportional to its lifetime, and anharmonic line-widths (lifetimes) increase (decrease) with increase in temperatures. The estimated lifetime can be calculated by $\tau=$ $1 / \pi c \Gamma$, where $c$ is the velocity of light and $\Gamma$ is the FWHM of the mode [23]. Phonon lifetime is $2.1 \mathrm{ps}$ at $80 \mathrm{~K}$ and $0.92 \mathrm{ps}$ at $440 \mathrm{~K}$ for the $F_{2 g}$ phonon of $\mathrm{CeO}_{2}$. The variation of the decay time of the $F_{2 g}$ phonon with increase in temperature has thus been estimated and is shown in Figure 6 . The discontinuity in slope observed at about $380 \mathrm{~K}$ is again detectable in the figure.

\section{Conclusions}

The behavior of the optical $F_{2 g}$ phonon of nanocrystalline cubic $\mathrm{CeO}_{2}$ as a function of temperature is investigated via Raman spectroscopy. The $F_{2 g}$ optical mode of $\mathrm{CeO}_{2}$ shows phonon softening with increasing temperature up to $440 \mathrm{~K}$. This phenomenon arises from dominating cubic phononphonon interaction and line-width broadening in the temperature range 80 to $440 \mathrm{~K}$. The temperature dependent data analysis reveals that this mode is a combination of quasiharmonic and true anharmonic terms wherein the latter has a higher contribution to the total anharmonicity. The three-phonon process dominated the four-phonon process. The lifetime of the first-order $F_{2 g}$ optical mode is found to decrease with increasing anharmonicity. An observable discontinuity in the slopes of the phonon softening and the line-width curves is seen at around $380 \mathrm{~K}$.

\section{Conflict of Interests}

The authors declare that there is no conflict of interests regarding the publication of this paper.

\section{Acknowledgments}

The authors are grateful to Director, NPL, for his constant encouragement and are thankful to CSIR for grant under Network Project NWP-45.

\section{References}

[1] S. P. S. Badwal and F. T. Ciacchi, "Oxygen-ion conducting electrolyte materials for solid oxide fuel cells," Ionics, vol. 6, no. 1-2, pp. 1-21, 2000.

[2] B. C. H. Steele, "Appraisal of $\mathrm{Ce}_{1-y} \mathrm{Gd}_{y} \mathrm{O}_{2-y / 2}$ electrolytes for ITSOFC operation at $500^{\circ}$ C," Solid State Ionics, vol. 129, no. 1, pp. 95-110, 2000.

[3] B. C. H. Steele, "Mass transport in materials incorporated in electrochemical energy conversion systems," Solid State Ionics, vol. 12, pp. 391-406, 1984.

[4] P. Wachter, "The magnetic susceptibility of CEO@ has shown to be paramagnetic," in Valence Instabilities, P. Wachter and H. Boppart, Eds., p. 145, North-Holland, Amsterdam, The Netherlands, 1982.

[5] P. Mishra and K. P. Jain, "Temperature-dependent Raman scattering studies in nanocrystalline silicon and finite-size effects," Physical Review B-Condensed Matter and Materials Physics, vol. 62, no. 22, Article ID 14790, 2000.

[6] G. Morell, W. Pérez, E. Ching-Prado, and R. S. Katiyar, "Anharmonic interactions in beryllium oxide," Physical Review B, vol. 53, no. 9, pp. 5388-5395, 1996.

[7] N. S. Yuksek, N. M. Gasanly, and A. Aydinli, "Anharmonic line shift and linewidth of the Raman modes in TlInS 2 layered crystals," Journal of Raman Spectroscopy, vol. 35, no. 1, pp. 55-60, 2004.

[8] P. Verma, S. C. Abbi, and K. P. Jain, "Raman-scattering probe of anharmonic effects in GaAs," Physical Review B, vol. 51, no. 23, Article ID 16660, 1995.

[9] Z. Dohcevic-Mitrovic, Z. V. Popović, and M. Šćepanović, "Anharmonicity effects in nanocrystals studied by Raman scattering spectroscopy," Acta Physica Polonica A, vol. 116, no. 1, pp. 36-41, 2009.

[10] M. J. Konstantinović, S. Bersier, X. Wang et al., "Raman scattering in cluster-deposited nanogranular silicon films," Physical Review B-Condensed Matter and Materials Physics, vol. 66, Article ID 161311, 2002.

[11] M. Balkanski, R. F. Wallis, and E. Haro, "Anharmonic effects in light scattering due to optical phonons in silicon," Physical Review B, vol. 28, no. 4, pp. 1928-1934, 1983.

[12] S. D. Pandey, K. Samanta, J. Singh, N. D. Sharma, and A. K. Bandyopadhyay, "Anharmonic behavior and structural phase transition in $\mathrm{Yb}_{2} \mathrm{O}_{3}$," AIP Advances, vol. 3, Article ID 122123, 2013.

[13] Z. V. Popović, Z. Dohčević-Mitrović, A. Cros, and A. Cantarero, "Raman scattering study of the anharmonic effects in $\mathrm{CeO}_{2-y}$ 
nanocrystals," Journal of Physics Condensed Matter, vol. 19, no. 49, Article ID 496209, 2007.

[14] J. E. Spanier, R. D. Robinson, F. Zhang, S.-W. Chan, and I. P. Herman, "Size-dependent properties of $\mathrm{CeO}_{2-y}$ nanoparticles as studied by Raman scattering," Physical Review B, vol. 64, no. 24, Article ID 245407, pp. 1-8, 2001.

[15] Z. D. Dohcevic-Mitrovic, M. Radović, M. Šćepanović et al., "Temperature-dependent Raman study of $\mathrm{Ce}_{0.75} \mathrm{Nd}_{0.25} \mathrm{O}_{2 \_} \delta$ nanocrystals," Applied Physics Letters, vol. 91, no. 20, Article ID 203118, 2007.

[16] S. Dogra, J. Singh, N. Dilawar Sharma et al., "Phase progression via phonon modes in lanthanide dioxides under pressure," Vibrational Spectroscopy, vol. 70, pp. 193-199, 2014.

[17] S. Dogra, N. D. Sharma, J. Singh, H. K. Poswal, S. M. Sharma, and A. K. Bandyopadhyay, "High pressure behavior of nanocrystalline $\mathrm{CeO}_{2}$ up to $35 \mathrm{GPa}$ : a Raman investigation," High Pressure Research, vol. 31, no. 2, pp. 292-303, 2011.

[18] T. Sato and S. Tateyama, "Temperature dependence of the linewidth of the first-order Raman spectrum for crystalline $\mathrm{CeO}_{2}$," Physical Review B, vol. 26, no. 4, pp. 2257-2260, 1982.

[19] W. H. Weber, K. C. Hass, and J. R. McBride, "Raman study of $\mathrm{CeO}_{2}$ : second-order scattering, lattice dynamics, and particlesize effects," Physical Review B, vol. 48, no. 1, pp. 178-185, 1993.

[20] V. Kanchana, G. Vaitheeswaran, A. Svane, and A. Delin, "Firstprinciples study of elastic properties of $\mathrm{CeO}_{2}, \mathrm{ThO}_{2}$ and $\mathrm{PoO}_{2}$," Journal of Physics Condensed Matter, vol. 18, no. 42, pp. 96159624, 2006.

[21] L. Gerward, J. Staun Olsen, L. Petit, G. Vaitheeswaran, V. Kanchana, and A. Svane, "Bulk modulus of $\mathrm{CeO}_{2}$ and $\mathrm{PrO}_{2}-$ an experimental and theoretical study," Journal of Alloys and Compounds, vol. 400, no. 1-2, pp. 56-61, 2005.

[22] M. Millot, R. T. Zaera, V. M. Sanjose, B. J. Marc, and J. Gonzalez, "Anharmonic effects in $\mathrm{ZnO}$ optical phonons probed by Raman spectroscopy," Applied Physics Letters, vol. 96, no. 15, Article ID 152103, 2010.

[23] K. Samanta, P. Bhattacharya, and R. S. Katiyar, "Temperature dependent E2 Raman modes in the ZnCoO ternary alloy," Physical Review B, vol. 75, no. 3, Article ID 035208, 2007.

[24] K. Kamali, T. R. Ravindran, C. Ravi, Y. Sorb, N. Subramanian, and A. K. Arora, "Anharmonic phonons of $\mathrm{NaZr}_{2}\left(\mathrm{PO}_{4}\right)_{3}$ studied by Raman spectroscopy, first-principles calculations, and $\mathrm{x}$ ray diffraction," Physical Review B, vol. 86, Article ID 144301, 8 pages, 2012.

[25] P. G. Klemens, "Anharmonic decay of optical phonons," Physical Review, vol. 148, no. 2, pp. 845-848, 1966. 

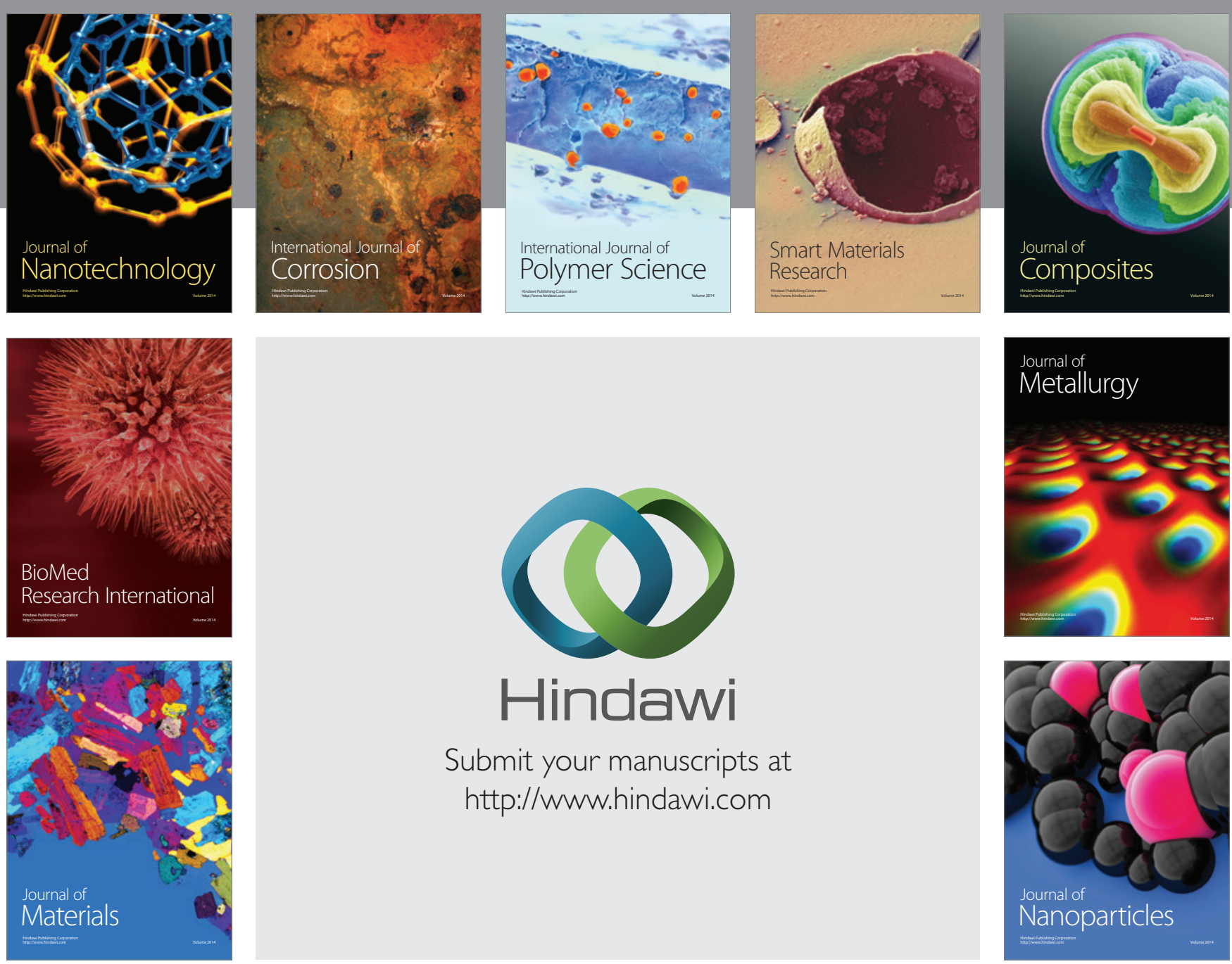

Submit your manuscripts at http://www.hindawi.com
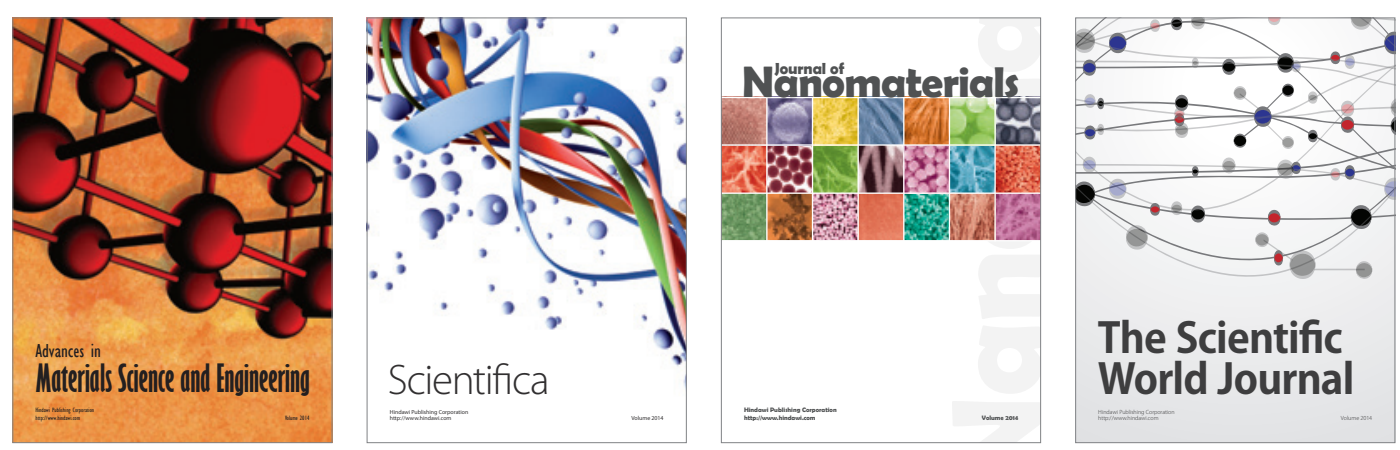

\section{The Scientific World Journal}
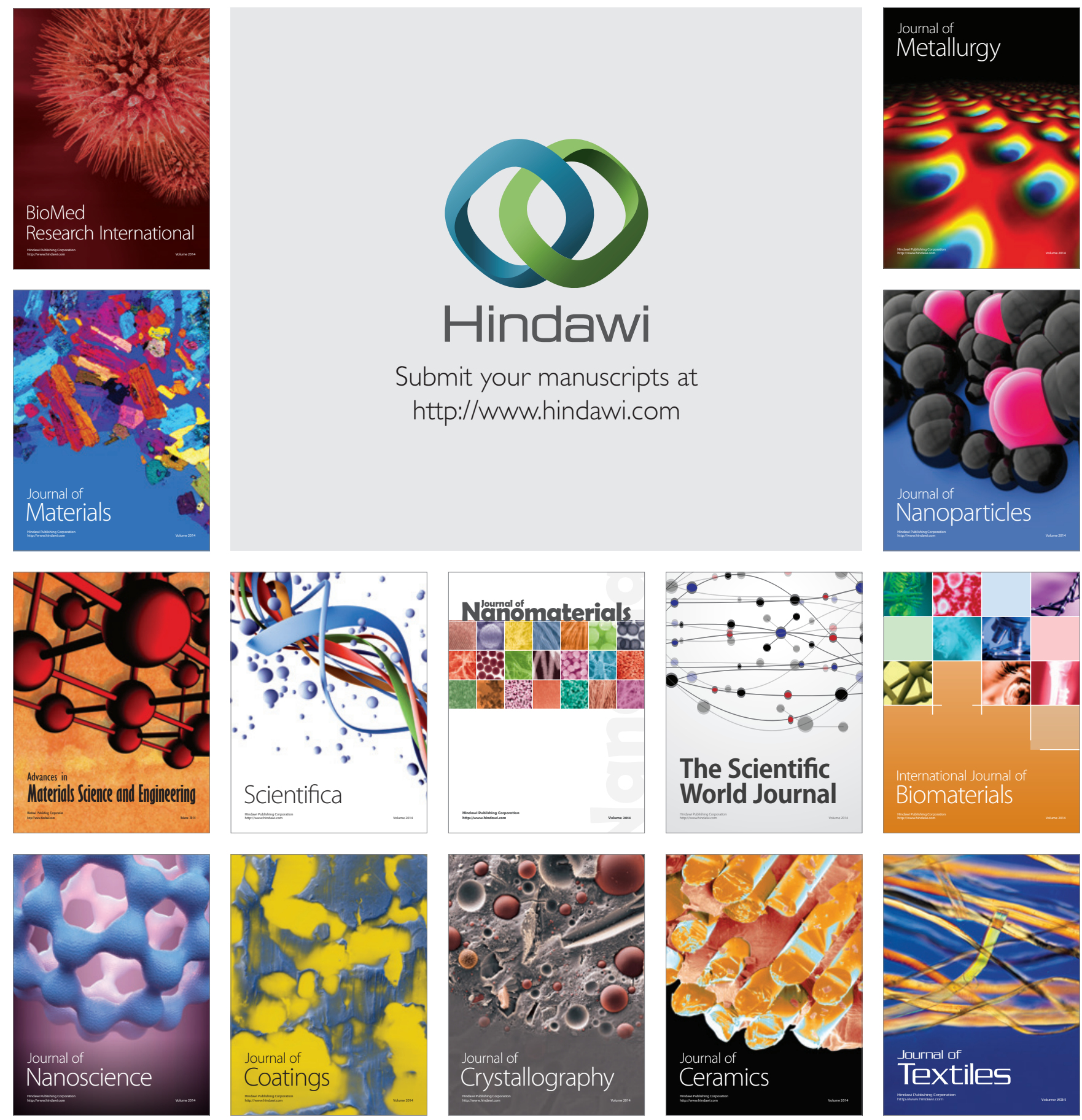